\title{
MEMS-based Tunable Fabry-Perot Filters for Adaptive Multispectral Thermal Imaging
}

\author{
Haifeng Mao, K. K. M. B. Dilusha Silva, Member, IEEE, Mariusz Martyniuk, Member, IEEE, \\ Jarek Antoszewski, John Bumgarner, Brett D. Nener, Senior Member, IEEE, John M. Dell, Member, IEEE \\ and Lorenzo Faraone, Fellow, IEEE
}

\begin{abstract}
This paper reports on a proof-of-concept MEMSbased Fabry-Perot filter that is capable of electrically tuning within the longwave infrared thermal imaging band of 8-12 $\mu \mathrm{m}$. The device employs a single-layer quarter-wavelength thick tensile germanium membrane for the suspended top mirror in order to achieve nanometre-scale as-released mirror flatness across an area of several hundred square microns without any extraneous stress management techniques. Mechanical and optical characterization of the tunable filters of various sizes are presented and compared. A $200 \mu \mathrm{m}$ dimension square filter is demonstrated with $<100 \mathrm{~nm}$ top mirror bowing and near-theoretical spectral characteristics across the entire tuning range of 8.5-11.5 $\mu \mathrm{m}$; namely, peak transmission above $80 \%$, FWHM of spectral passband of approximately $500 \mathrm{~nm}$, and out-of-band rejection greater than 40:1. Optical modelling shows that this filter can achieve a pixel-to-pixel transmission peak wavelength variation of less than $1.2 \%$ across the entire $200 \mu \mathrm{m} \times 200 \mu \mathrm{m}$ optical imaging area. These results exceed the optical performance requirements for passive multispectral thermal imaging applications based on large-area focal plane arrays. In comparison, the 500- $\mu \mathrm{m}$ and $1000-\mu \mathrm{m}$ dimension filters are shown to exhibit significant mirror bowing with actuation and, thus, for a pixel-to-pixel transmission peak wavelength non-uniformity of $<\mathbf{4} \%$, demonstrate narrower usable spectral tuning ranges of 9.3-11.4 $\mu \mathrm{m}$ and 10.3-11.3 $\mu \mathrm{m}$, respectively.
\end{abstract}

Index Terms-Fabry-Perot, longwave infrared, LWIR, microelectromechanical systems, MEMS, multispectral, thermal imaging.

\section{INTRODUCTION}

$\mathbf{M}$ ULTISPECTRAL thermal imaging systems capture completely passive thermal images of emitted radiation over multiple discrete wavelength bands of interest within the longwave infrared (LWIR: $8-12 \mu \mathrm{m}$ ) spectral range. The resulting image is a direct result of any emissivity and/or temperature differences within the field of view. Such systems can extract spectral signatures of objects of interest, and thus provide enhanced detection and discrimination of targets in clutter in comparison to single-band imaging systems. The utility of LWIR multispectral thermal imaging systems is well established in a wide variety of applications, ranging from gas and volatile organic compound diagnostics [1]-[3], mineral mapping [4], [5], and global atmospheric temperature profile monitoring [6], [7]. Conventional multispectral thermal imagers select spectral bands by means of a series of bandpass interference filters mounted on a motorized filter wheel, and are characterized by significant size and cost, as well as mechanical complexity. The number of wavelength bands available in a filter wheel based imager is limited by the number of slots available in the filter wheel, and the slow mechanical switching speed of the filter wheel gives rise to slow spectral switching speed and, thus, slow imaging rate. All these disadvantages have hindered the widespread adoption of the technology. The approach chosen in this paper is to develop a micro-electromechanical systems (MEMS) based electrostatically tuned Fabry-Perot filter that is suitable for hybridization with a 2-D imaging focal plane array (FPA). Such a module would be able to provide an efficient LWIR adaptive multispectral thermal imaging system with significantly reduced size, weight and power (SWaP) requirements suitable for field-portable and airborne applications.

A Fabry-Perot filter consists of two parallel mirrors separated by an optical cavity. The mirrors are typically distributed Bragg reflectors composed of alternating quarter-wave thick layers of high-refractive-index and low-refractive-index materials. Ignoring phase changes on mirror reflection, the optical transmission of the filter is maximized at the resonant wavelength that is exactly double the optical cavity length, when operating in first-order [8]. For other wavelengths, the optical transmission falls off rapidly away from the peak resonance value, creating a spectrally isolated transmission pass-band. Using an electrical stimulus to control the mirror separation, the transmission pass-band can be swept over a range of wavelengths, realizing an electrically tunable optical filter. The critical spectral parameters of a tunable FabryPerot filter include the wavelength tuning range, the peak transmission, the full-width at half-maximum (FWHM) of the transmission peak, the out-of-band rejection (transmission maximum-to-minimum ratio), and the pixel-to-pixel transmission peak wavelength uniformity across the optical imaging area of the filter. Table I summarises the target values for these critical metrics in a viable multispectral thermal imaging system. The specifications of $>50 \%$ peak transmission and $>10$ : 1 out-of-band rejection are based on the requirements of an industry-related project that is currently being undertaken, and the $0.5-1 \mu \mathrm{m}$ FWHM is required in order to discriminate the LWIR spectral signatures of different targets [1]-[5]. The $<4 \%$ peak wavelength variation requirement is proposed in order to ensure that, even at $12 \mu \mathrm{m}$, the maximum difference in the central peak wavelength between different imaging pixels is less than $480 \mathrm{~nm}$, which is less than the filter spectral resolution of $500 \mathrm{~nm}$.

Whilst MEMS-based LWIR Fabry-Perot filters have been previously reported [9]-[13], these published works concentrate on achieving high spectral resolution by incorporating 
TABLE I

PERFormance ReQuirements of TunABle Filters For LWIR MultisPECTRAL THERMAL IMAGING APPLICATIONS

\begin{tabular}{lll}
\hline \hline Parameter & Value & Comment \\
\hline Tuning range (within) & $8-12 \mu \mathrm{m}$ & Complete thermal band \\
\hline Peak transmission & $>50 \%$ & $\begin{array}{l}\text { Optical throughput for high } \\
\text { signal-to-noise ratio }\end{array}$ \\
\hline Full-width at half-maximum & $0.5-1 \mu \mathrm{m}$ & $\begin{array}{l}\text { Optical throughput and } \\
\text { wavelength discrimination }\end{array}$ \\
\hline Out-of-band rejection & $>10: 1$ & Wavelength discrimination \\
\hline Peak wavelength variation & $<4 \%$ & $\begin{array}{l}\text { Pixel-to-pixel wavelength } \\
\text { uniformity across a 2-D FPA }\end{array}$ \\
\hline
\end{tabular}

highly reflective multilayer Bragg mirrors. These studies include Teledyne [9] with $120 \mathrm{~nm}$ FWHM, and InfraTec [10], [11] who report a $200 \mathrm{~nm}$ FWHM using multilayer Ge/ZnSbased mirrors. Any top mirror deformation arising from stress mismatch between different mirror layers will degrade filter transmission [14], [15], and in particular, cause pixel-to-pixel transmission peak wavelength non-uniformity, and therefore, complicates fabrication of these filters. Mirror stress gradients can be balanced by tailoring the layer stress conditions [9], but this requires extensive material characterization and stringent process control to minimize run-to-run processing variations, which can make it difficult to achieve an acceptable yield. To control stress gradients induced mirror deformation, very thick, mechanically stiff carrier wafers have also been used [10], [11]. However, this increases the chip size considerably. In comparison, VTT [12], [13] has realized a LWIR filter with $110 \mathrm{~nm}$ FWHM based on symmetrical Si/air/Si mirrors, which are not subject to any film stress mismatch issue. However, this filter requires an undesirable high-temperature annealing process to control the Si film stress in order to produce flat mirrors.

In this paper, we report on a thin-film surfacemicromachined MEMS-based tunable LWIR Fabry-Perot filter with a quarter-wavelength thick tensile-strained Ge membrane as the suspended top mirror. The reported tunable filter is capable of achieving near-perfect mirror flatness after release without the need for any sophisticated stress management techniques, and exhibits spectral characteristics that exceed the performance requirements for multispectral thermal imaging applications, as summarized in Table I.

\section{Structural Design}

The Fabry-Perot filter structure is shown in Fig. 1, and consists of a single quarter-wavelength thick Ge layer as the suspended top mirror, with a fixed quarter-wave Ge/ZnS/Ge/ZnS bottom mirror on a silicon substrate. A quarter-wavelength thick $\mathrm{ZnS}$ layer is deposited on the backside of the substrate as an anti-reflection coating to reduce backside reflections at the substrate-air interface. The top Ge mirror layer extends all the way out to the outer perimeter of the polyimide, which serves as the MEMS structural support. The advantage of this design is that any tensile stress inherent in the $\mathrm{Ge}$ layer will tend to stretch the whole suspended membrane,

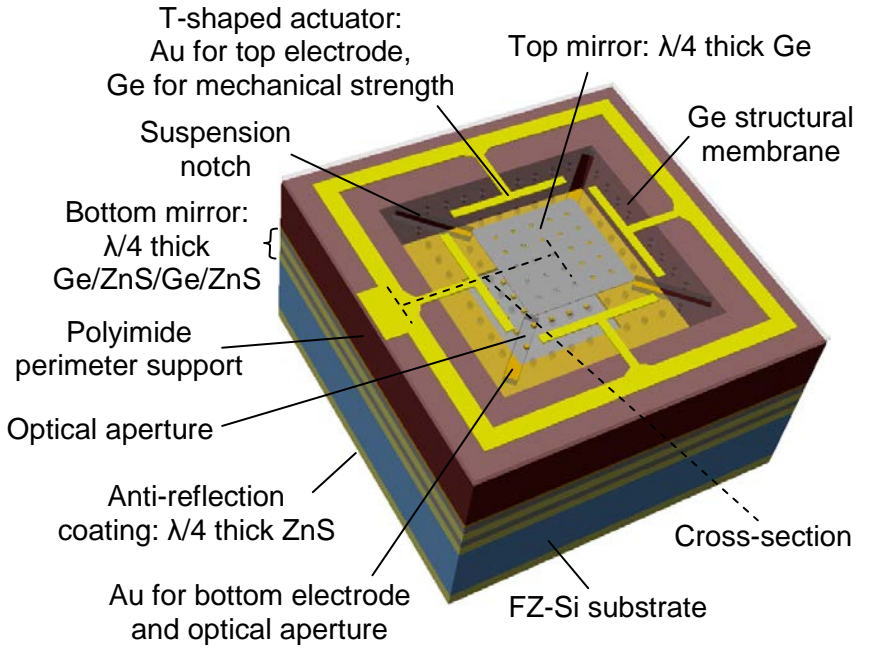

(a)

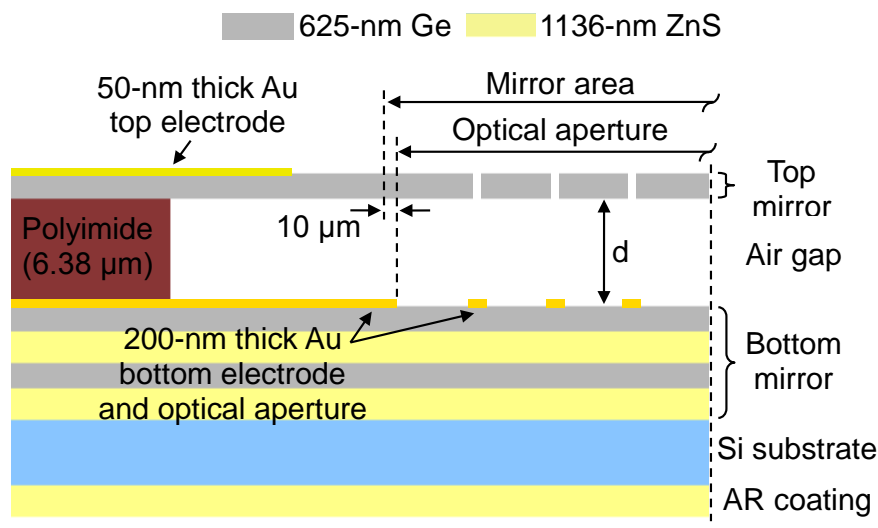

(b)

Fig. 1. (a) Graphical depiction of the MEMS-based tunable LWIR FabryPerot filter. The top membrane, except for the central mirror region, is drawn semi-transparent for illustration purposes. (b) Cross-section depiction of the filter. Layer thicknesses shown in the figure are the target values.

thus aiding in flattening the upper mirror and eliminating the need for any stress management. Driving Au electrodes are deposited around the central mirror which, in combination with the underlying bottom Au electrode, move the top mirror closer to the bottom mirror during electrostatic actuation. Diagonally arranged notches at the corners of the top mirror actuation structure serve to release the convergence of stress from two orthogonal directions. The notches also reduce the spring stiffness of the membrane and allow actuation at lower voltages. An array of 5- $\mu \mathrm{m}$ diameter holes are perforated in the membrane, which facilitates the sacrificial layer etching process and results in a short release time that prevents any deterioration of the Ge membrane due to over-exposure to the reactive gas plasma used during preferential removal of the underlying polyimide sacrificial layer. These etch holes are only included in this proof-of-concept filter design, and can be removed in any future development. The underlying bottom $\mathrm{Au}$ electrode serves as an optical shield for stray light, and is designed with an aperture under the mirror area that includes an array of $\mathrm{Au}$ dots to mask the optical path of the array of etch holes in the top Ge membrane. 


\section{Optical Modelling}

The Ge and ZnS mirror layers in the proposed LWIR FabryPerot filter were designed to be quarter-wavelength thick at the wavelength of $10 \mu \mathrm{m}$. Based on our experimental values of refractive indices $(n=4.0$ for Ge and $n=2.2$ for $\mathrm{ZnS}$ [16]), the quarter-wave thicknesses of the Ge and $\mathrm{ZnS}$ layers were determined to be $625 \mathrm{~nm}$ and $1136 \mathrm{~nm}$, respectively.

For such an asymmetric optical filter, with the top mirror consisting of a single quarter-wave optical layer and being less reflective than the bottom mirror, a relatively wide spectral bandwidth is expected because of the resulting low cavity finesse. Fig. 2 shows the ideal optical transmission of the designed filter, calculated using the optical matrix method [17] for various cavity lengths $d$. Due to the fact that Ge and $\mathrm{ZnS}$ have very low dispersion in the LWIR band [18], [19], in the optical modelling the refractive indices of $\mathrm{Ge}$ and $\mathrm{ZnS}$ layers were assumed to be constant over the entire 8-12 $\mu \mathrm{m}$ range, i.e., $n=4.0$ for $\mathrm{Ge}$ and $n=2.2$ for $\mathrm{ZnS}$. These calculated spectra indicate that the proposed filter is capable of achieving peak transmission higher than $80 \%$ and a spectral pass-band (FWHM) of $0.5 \mu \mathrm{m}$ across the entire 8-12 $\mu \mathrm{m}$ tuning range. As can be seen in Fig. 2, the filter also features an out-of-band light transmission of approximately $2 \%$, which corresponds to an out-of-band rejection for the filter that is better than 40:1. These figures exceed the optical requirements listed in Table I, and demonstrate that the proposed LWIR Fabry-Perot filter design is a viable solution, provided that the flatness of the suspended mirror results in a uniform optical cavity that can meet the peak wavelength uniformity requirement. Fig. 2 also shows that except for the case of the central design wavelength of $10 \mu \mathrm{m}$, transmission peaks of the filter never occur exactly at wavelengths that are double the optical cavity length, which is a direct consequence of optical phase changes on reflection from the quarter-wave mirror surface [20].

\section{Tunable Filter Fabrication}

Prior to filter fabrication, deposition conditions for $\mathrm{Ge}$ and $\mathrm{ZnS}$ thin films that are compatible with microfabrication processes were established [16]. Durable Ge films were deposited via electron-beam evaporation at room temperature, and durable $\mathrm{ZnS}$ films were deposited via thermal evaporation at a substrate temperature of $150{ }^{\circ} \mathrm{C}$. In addition, appropriate silicon substrates were chosen in terms of longwave infrared optical transparency. The strong absorption at $9 \mu \mathrm{m}$ due to excessive oxygen content renders standard Czochralski (CZ) silicon substrates unsuitable for LWIR applications [21]. In comparison, float-zone (FZ) silicon wafers contain negligible amounts of oxygen and exhibit excellent LWIR transparency, and were therefore used as supporting substrates for LWIR filter fabrication.

The Fabry-Perot filters were fabricated using thinfilm surface micromachining. Initially, a quarter-wave $\mathrm{Ge} / \mathrm{ZnS} / \mathrm{Ge} / \mathrm{ZnS}$ bottom mirror stack was deposited on the FZ silicon substrate. A quarter-wavelength thick $\mathrm{ZnS}$ antireflection coating was then deposited on the backside of the substrate. This was followed by thermal deposition of a 200$\mathrm{nm} \mathrm{Au}$ layer on the bottom mirror, and patterned using liftoff. This metal layer acted as the bottom electrode as well

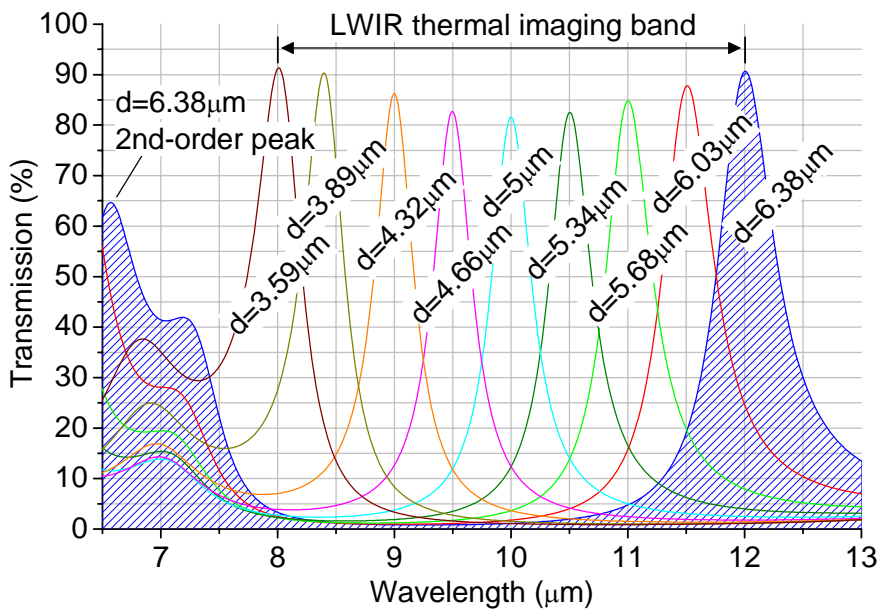

Fig. 2. Modelled transmission spectra of the MEMS-based LWIR Fabry-Perot filter shown in Fig. 1 for various cavity lengths $d$, neglecting mirror defects such as tilt or bowing.

as an optical shield to eliminate stray light leakage around the filter. The opening in this Au layer defines the optical aperture of the filter, which was $20 \mu \mathrm{m}$ laterally smaller than the mirror in order to avoid unintended stray light due to any misalignment errors. Importantly, this $\mathrm{Au}$ layer includes an array of circular micropatterns which are aligned with the etch hole array perforated at a later processing stage in the top mirror, and thus will shield any stray light transmitted through the etch holes. To achieve a $6.38 \mu \mathrm{m}$ thick sacrificial layer required for resonance at $12 \mu \mathrm{m}$ LWIR wavelength, the polyimide was spun on the wafer using a multiple-coating technique. Measurements showed that the coating thicknesses of the polyimide layer were $5.93 \mu \mathrm{m}, 5.89 \mu \mathrm{m}$ and 5.82 $\mu \mathrm{m}$ for the $200-\mu \mathrm{m}, 500-\mu \mathrm{m}$ and $1000-\mu \mathrm{m}$ dimension filters on three separate samples, respectively. A quarter-wavelength thick Ge membrane layer was then coated onto the sample. Subsequently, a 50-nm Au layer was deposited using thermal evaporation and lift-off, in order to form the top electrodes. Diagonal notches and the hole array were then etched in the top $\mathrm{Ge}$ membrane using a $\mathrm{CF}_{4} / \mathrm{O}_{2}$ plasma. The etch hole array defines the suspended membrane area and facilitates the subsequent filter release process. The upper mirror membrane was released by etching the sacrificial polyimide through the array of holes using an $\mathrm{O}_{2}$ plasma. The remaining polyimide around the filter perimeter serves as a structural support for the suspended top mirror. Fig. 3a illustrates the structural layout and dimensions of the fabricated filters, and Fig. 3b shows a scanning electron microscope (SEM) image of a $500-\mu \mathrm{m}$ dimension filter.

\section{Device Performance}

\section{A. Electromechanical characterization}

In order to study the electromechanical behaviour of the fabicated filters, an optical surface profilometer was used to obtain 3D topographical images (height maps) of the filters during actuation. The deflection profiles of the free-standing membranes for the three different-sized filters were extracted along the line "AB" as indicated in Fig. 3b, and are presented 


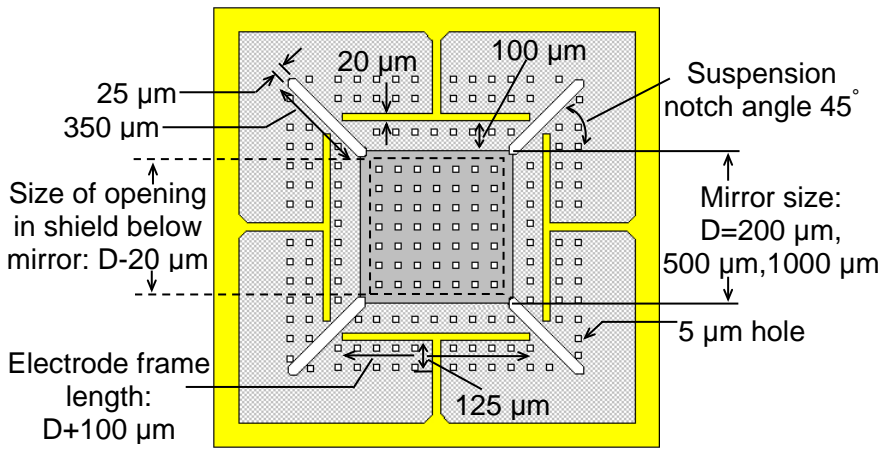

(a)

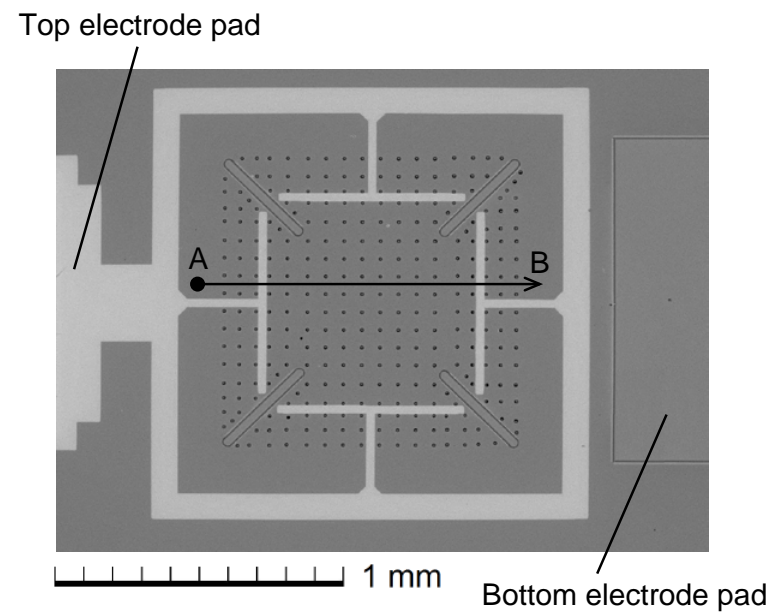

(b)

Fig. 3. (a) Structural layout and dimensions of the MEMS-based tunable LWIR Fabry-Perot filters. The central mirror region is highlighted and the size of the etch holes is exaggerated for illustration purposes only. The optical aperture in the shield below the mirror can be seen in Fig. 1a. (b) Scanning electron microscope (SEM) image of the fabricated 500- $\mu$ m dimension filter The line "AB" indicates the location of the mirror surface line profiles presented in Fig. 4.

in Fig. 4 for various actuation voltages. The surface line profiles reveal the displacement and the bowing magnitude of the central mirror region, and the location of the anchor plane and the top metal electrode.

As evident from the insets of Fig. 4, the central mirrors of the three un-actuated filters exhibited near-perfect flatness, with bowing on the nanometer scale across the entire optical area. As the actuation voltage increases, top mirror bowing was developed. We observed the largest bowing magnitudes of $100 \mathrm{~nm}$ at $160 \mathrm{~V}$ for the $200-\mu \mathrm{m}$ filter; $400 \mathrm{~nm}$ at $135 \mathrm{~V}$ for the $500-\mu \mathrm{m}$ filter; and $550 \mathrm{~nm}$ at $85 \mathrm{~V}$ for the $1000-\mu \mathrm{m}$ filter. Overall, larger filters require lower voltages to actuate but develop more significant mirror bowing with actuation in comparison to smaller filters. Any additional increase in the actuation voltage above these values resulted in mechanical failure of the membrane, which occurred before the expected electrical snap-down at one-third of the unactuated electrode separation. We believe that this phenomenon is most likely due to severe overall tensile forces developed in the Ge membrane during actuation. Further investigation is required to clarify this issue. Moreover, it is noteworthy to observe in Fig. 4 that all filters exhibited negligible mirror tilt over the entire tuning range, although mirror bowing is commonplace during filter actuation.

\section{B. Optical characterization}

Fourier transform infrared spectroscopy (FTIR) was used to measure the total transmission of the filters as a function of actuating voltage. For calibration purposes, a set of reference samples consisting of $180-\mu \mathrm{m}, 480-\mu \mathrm{m}$ and $980-\mu \mathrm{m}$ dimension through-wafer square holes (identical to the optical apertures of the filters) were fabricated by deep reactive-ion etching $\mathrm{Si}$ substrates using a $\mathrm{SF}_{6}$ plasma and $200 \mathrm{~nm}$ thick $\mathrm{Al}$ as the etching mask. These samples, along with the $\mathrm{Al}$ optical masking layers, acted as the light shield for optical reference measurements. The intensity of light transmitted through the fabricated openings was measured and multiplied by a correction factor to account for the area of the etch hole array perforated in the top quarter-wave thick Ge mirror, thereby yielding the reference spectrum for each filter size. This correction factor was derived by subtracting the total area of the etch hole array from the optical aperture area, and then dividing this value by the optical aperture area. The transmitted light intensity through the filters was subsequently recorded as a function of actuating voltage and normalized by the respective reference spectrum to yield the transmission tuning spectra for each filter size.

The experimentally measured tuning spectra for the filters of three different sizes are presented in Fig. 5a using data points. The solid lines in Fig. 5a represent the transmission spectra calculated using the following procedure: firstly, based on the $3 \mathrm{D}$ topographical filter images measured from an optical profilometer, the bowed filters were segmented into a set of closely spaced $2 \mu \mathrm{m} \times 2 \mu \mathrm{m}$ elements of ideal filters. Subsequently, the transmission of each ideal filter element was calculated using the optical matrix model [17] and weighted by each element area relative to the total mirror optical area. Finally, the total transmission of the filters was obtained by summing the weighted transmission of all the individual elements. As can be seen in Fig. 5a, a strong agreement is demonstrated between the modelled and measured results, validating that this modelling approach is an effective tool for calculating the transmission spectrum of Fabry-Perot filters in the presence of mirror bowing. In this paper, this modelling approach was also employed to calculate the on-pixel transmission spectra of the filters over a $20-\mu \mathrm{m}$ pixel area at the centre and edge of the mirror optical area, in order to evaluate the transmission peak wavelength uniformity across the optical imaging area of the filters, and the results are shown in Fig. 5b.

As can be seen in Fig. 5a for the 200- $\mu$ m dimension filter, the measured wavelength tuning range was observed to be 8.5-11.46 $\mu \mathrm{m}$ and was obtained using a maximum voltage of $160 \mathrm{~V}$. This wavelength tuning range accounts for $75 \%$ of the complete thermal imaging band of $8-12 \mu \mathrm{m}$, and to date is the widest tuning range reported in the literature for a MEMS-based LWIR Fabry-Perot filter. The 200- $\mu$ m filter exhibited consistent near-ideal transmission characteristics over the entire tuning range, including a high peak transmission in the range of $80-87 \%$, a FWHM value of 


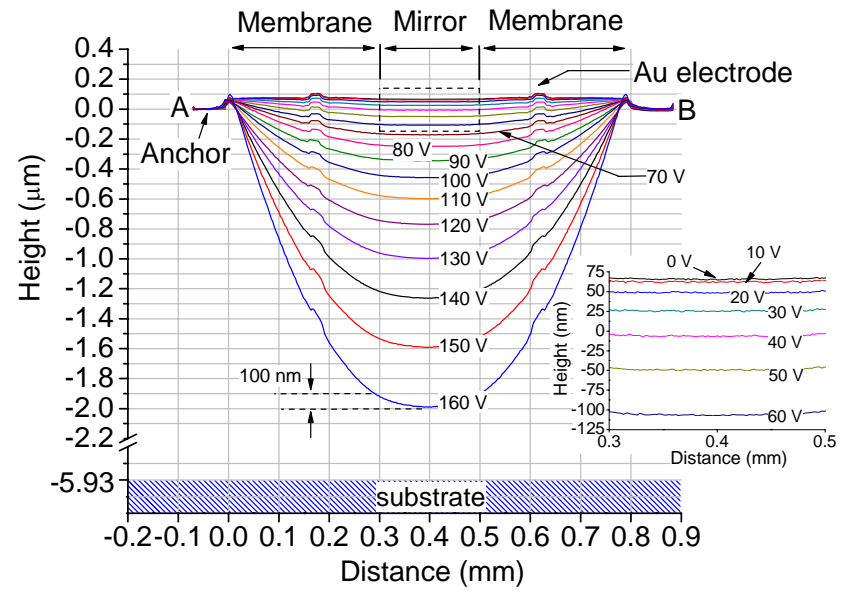

(a)

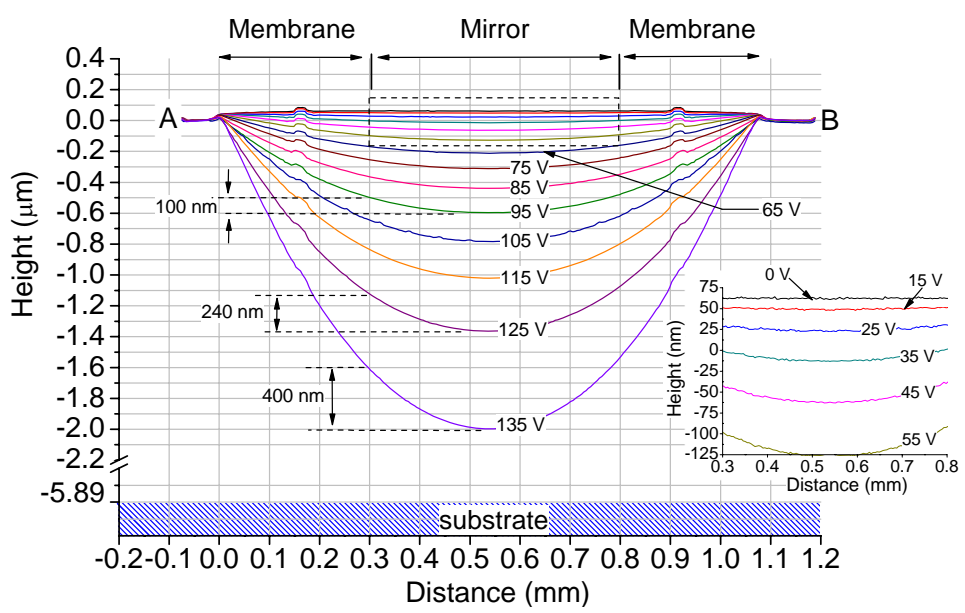

(b)

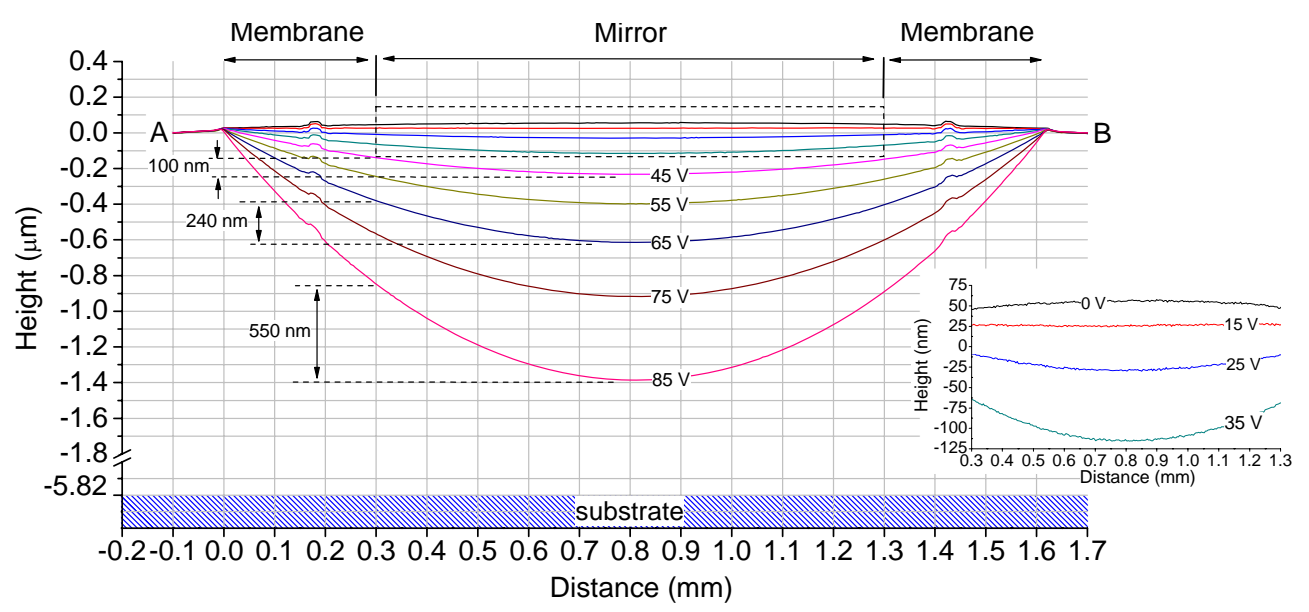

(c)

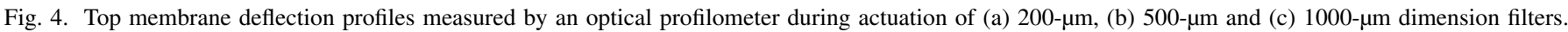
Insets show profiles of the mirror optical area on an expanded scale at low actuation voltages.

$\sim 500 \mathrm{~nm}$ and an out-of-band rejection better than 40:1. Moreover, the modelling results in Fig. $5 \mathrm{~b}$ show that this $200-\mu \mathrm{m}$ dimension filter is capable of achieving a pixel-topixel transmission peak wavelength uniformity of better than $1.2 \%$ across a $200 \mu \mathrm{m} \times 200 \mu \mathrm{m}$ optical imaging area. These superior spectral parameters are a direct consequence of the achieved level of suspended top mirror flatness, and exceed the optical performance requirements listed in Table I, resulting in the 200- $\mu \mathrm{m}$ dimension tunable filters being most suitable for use in multispectral thermal imaging applications.

The significant degradation of the overall filter transmission peak and FWHM observed in the tuning spectra of the 500$\mu \mathrm{m}$ and $1000-\mu \mathrm{m}$ dimension filters correlates well with the mirror bowing trends observed in Fig. 4 for these two filters. In contrast, it is noted that in a 2-D imaging array the individual pixels only 'see' a very small part of the Fabry-Perot filter (in our case, an area of $20 \mu \mathrm{m} \times 20 \mu \mathrm{m}$ ). Thus, any largearea mirror bowing will not result in a transmission peak decrease or FWHM broadening on the pixel level. However, it will cause a variability of the optical cavity length, which will translate into a pixel-to-pixel variation of transmission peak wavelength across the imaging array. As can be seen in Fig. 5b, the larger 500- $\mu \mathrm{m}$ and $1000-\mu \mathrm{m}$ dimension filters can achieve ideal on-pixel transmission characteristics over the entire tuning range regardless of magnitudes of top mirror bowing; namely, a peak transmission higher than $80 \%$, a FWHM of approximately $500 \mathrm{~nm}$, and an out-of-band rejection better than 40:1, which exceed the optical requirements for multispectral thermal imaging applications. However, for both filters, the required pixel-to-pixel peak wavelength variability $(\Delta \lambda / \lambda<4 \%)$ can only be achieved over a limited actuation range; namely, 9.3-11.4 $\mu \mathrm{m}$ for the 500- $\mu \mathrm{m}$ dimension filter with an actuation voltage of less than $125 \mathrm{~V}$, which accounts for $52 \%$ of the complete thermal imaging band, and 10.3-11.3 $\mu \mathrm{m}$ for the $1000-\mu \mathrm{m}$ dimension filter with an actuation voltage of less than $65 \mathrm{~V}$, which accounts for $25 \%$ of the thermal imaging band. These wavelength ranges represent the usable spectral tuning ranges for these two filters in order to meet the performance specifications listed in Table I. By correlating these observations with the mirror bowing measurements in Fig. 4, it can be concluded that in order to achieve the required peak wavelength uniformity specification, top mirror bowing needs to be limited to within $240 \mathrm{~nm}$ over the entire optical area. Mirror bowing beyond this level will result in consider- 


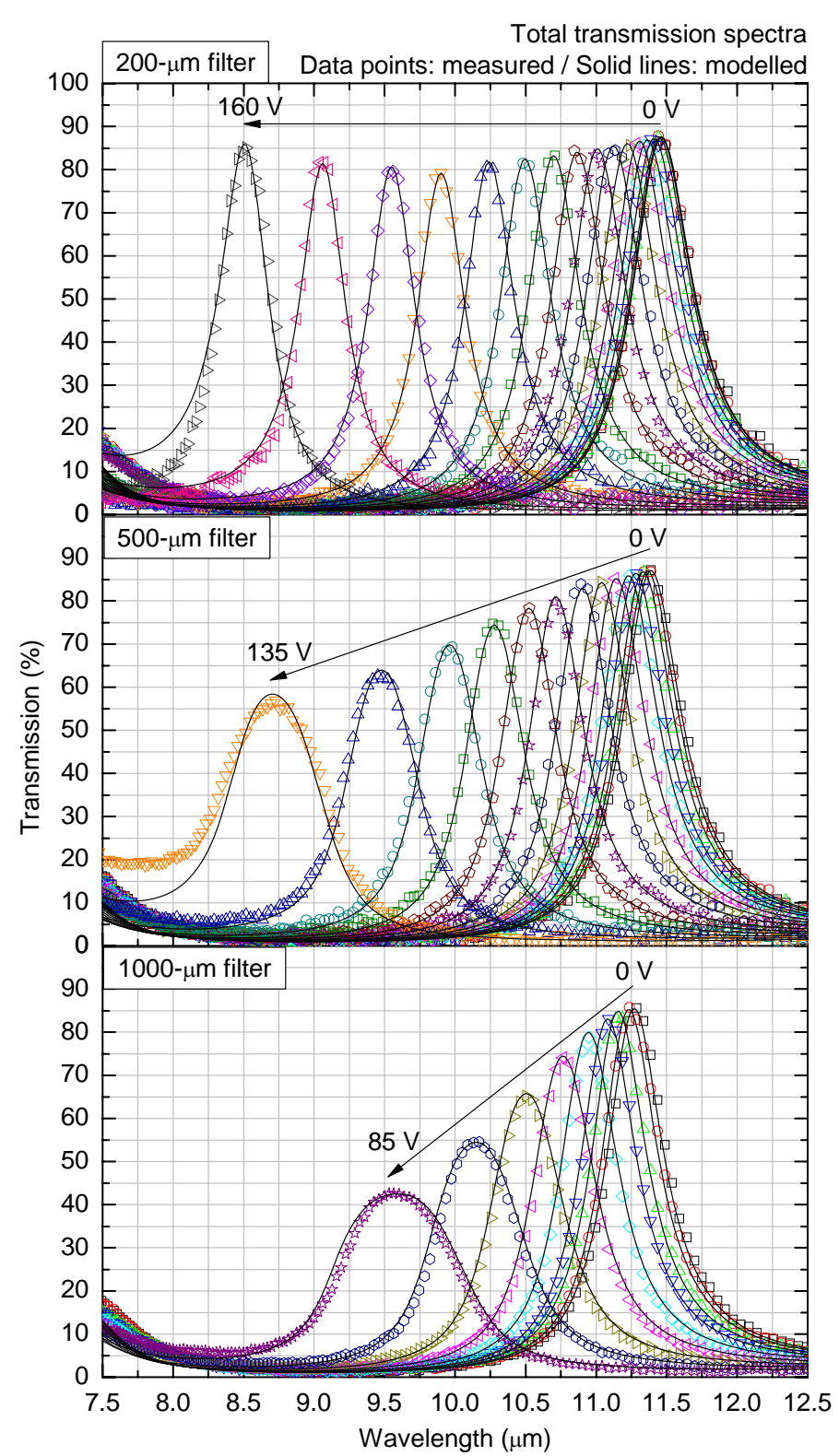

(a)
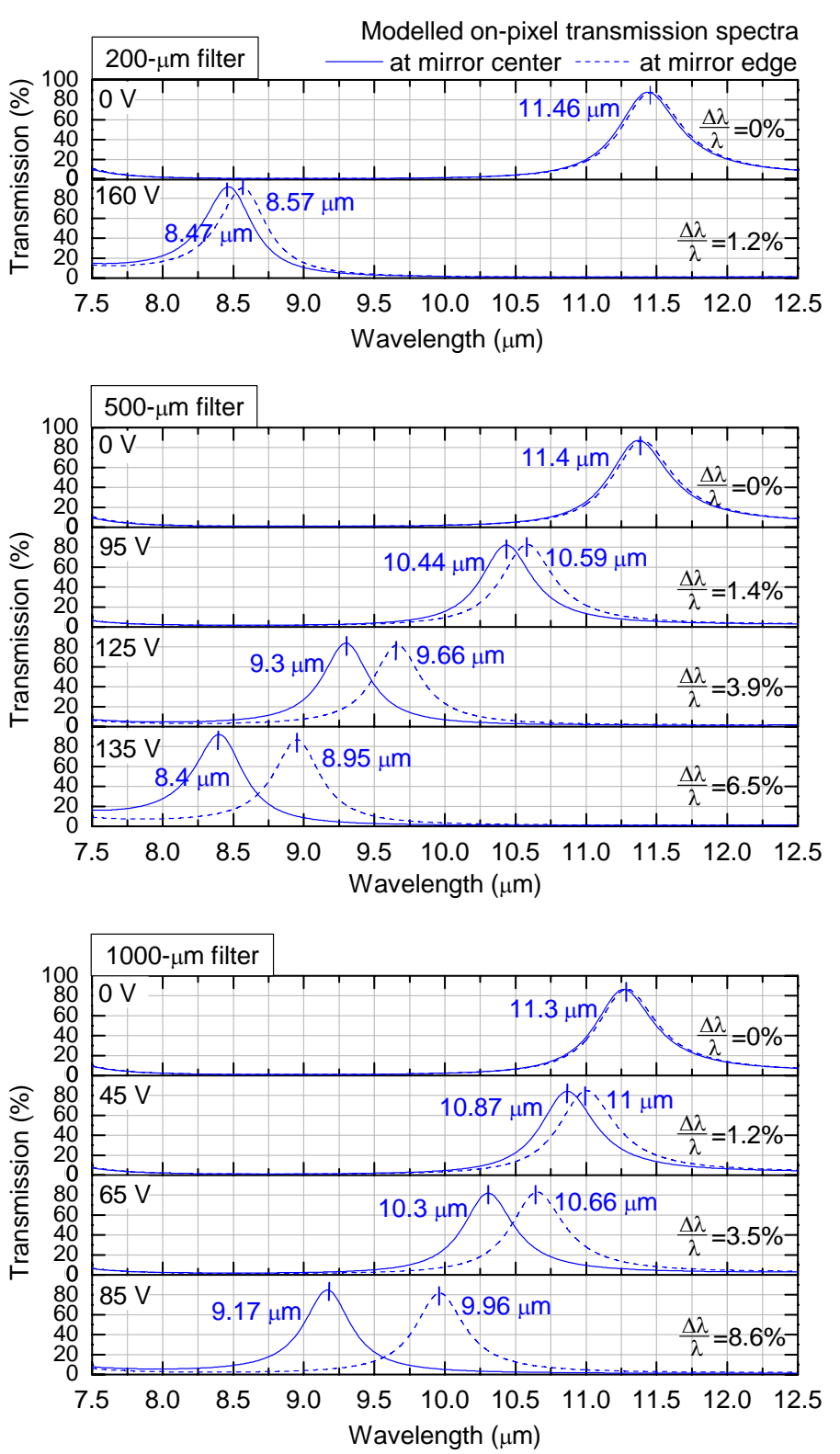

(b)

Fig. 5. (a) Total transmission measured by FTIR (data points) during actuation of $200-\mu \mathrm{m}, 500-\mu \mathrm{m}$ and $1000-\mu \mathrm{m}$ dimension filters, and the transmission calculated from the optical modelling described in the text (solid lines). (b) Modelled on-pixel filter transmission over a $20 \mu \mathrm{m} \times 20 \mu \mathrm{m}$ pixel area at the centre and edge of the mirror for various actuation voltages. $\lambda$ is the peak wavelength at the centre of the mirror, and $\Delta \lambda$ is the separation between the peak wavelengths at the centre and edge of the mirror.

able pixel-to-pixel peak wavelength non-uniformity, rendering the filters unusable for multispectral thermal imaging applications. For the 500- $\mu \mathrm{m}$ dimension filter, as the actuation voltage is increased to $135 \mathrm{~V}$ and the bowing of the mirror over the optical area is correspondingly increased to $400 \mathrm{~nm}$, pixel-topixel transmission peak wavelength variation across the filter increases to $6.5 \%$. For the $1000-\mu \mathrm{m}$ dimension filter, at the maximum actuation voltage of $85 \mathrm{~V}$ and the corresponding mirror bowing of $550 \mathrm{~nm}$, this value reaches $8.6 \%$, more than double the maximal specified value. Table II summarizes the centre-to-edge bowing magnitudes of the suspended top mirrors, the wavelength tuning ranges, and the pixel-to-pixel transmission peak wavelength variations of the three filters for different actuation voltage ranges.

\section{CONCLUSIONS}

MEMS-based long-wavelength infrared (LWIR) FabryPerot optical filters are essential for the future realization of compact, low-cost and efficient multispectral thermal imaging systems for field-portable and airborne applications. Successful development of MEMS-based tunable LWIR FabryPerot filters is technologically challenging due to the stress induced bowing of suspended mirrors compounded by the requirement to incorporate significant stress control mechanisms. This paper has reported the realization of a MEMSbased tunable LWIR Fabry-Perot filter, employing a single- 
TABLE II

TOP MIRROR BOWING MAGNITUDES, WAVELENGTH TUNING RANGES AND PIXEL-TO-PIXEL PEAK WAVELENGTH VARIATIONS OF THE 200- $\mu$ m, 500- $\mu \mathrm{m}$ AND 1000- $\mu$ m DIMENSION FILTERS FOR DIFFERENT ACTUATION VOLTAGE RANGES

\begin{tabular}{|c|c|c|c|c|c|c|c|}
\hline & \multirow{2}{*}{$\frac{200-\mu \mathrm{m} \text { filter }}{0-160 \mathrm{~V}}$} & \multicolumn{3}{|c|}{$500-\mu \mathrm{m}$ filter } & \multicolumn{3}{|c|}{$1000-\mu \mathrm{m}$ filter } \\
\hline & & $0-95 \mathrm{~V}$ & $95-125 \mathrm{~V}$ & $125-135 \mathrm{~V}$ & $0-45 \mathrm{~V}$ & $45-65 \mathrm{~V}$ & $65-85 \mathrm{~V}$ \\
\hline Mirror bowing (nm) & $0-100$ & $0-100$ & $100-240$ & $240-400$ & $0-100$ & $100-240$ & $240-550$ \\
\hline Wavelength tuning range ( $\mu \mathrm{m}$, at mirror centre) & $11.46-8.47$ & $11.4-10.44$ & $10.44-9.3$ & $9.3-8.4$ & $11.3-10.87$ & $10.87-10.3$ & $10.3-9.17$ \\
\hline Peak wavelength variation & $0-1.2 \%$ & $0-1.4 \%$ & $1.4-3.9 \%$ & $3.9-6.5 \%$ & $0-1.2 \%$ & $1.2-3.5 \%$ & $3.5-8.6 \%$ \\
\hline
\end{tabular}

layer quarter-wavelength thick tensile-strained Ge membrane as the suspended top mirror that requires no additional stress management to achieve nanometre-scale as-released mirror flatness. The fabricated square filters of $200 \mu \mathrm{m}$ in dimension showed a continuous wavelength tuning range of $8.5-11.46 \mu \mathrm{m}$ under a maximum actuation voltage of $160 \mathrm{~V}$, demonstrating $<100 \mathrm{~nm}$ mirror bowing over the entire tuning range and exhibiting spectral characteristics that exceed the required specifications for multispectral thermal imaging applications; namely, a peak transmission higher than $80 \%$, a spectral bandwidth of approximately $500 \mathrm{~nm}$, an out-of-band rejection better than 40:1. It has also been shown that the 200- $\mu$ m dimension filter can achieve excellent pixel-to-pixel transmission peak wavelength uniformity during the entire actuation, with a peak wavelength variation of less than $1.2 \%$ across a $200 \mu \mathrm{m} \times$ $200 \mu \mathrm{m}$ optical area. In comparison, the 500- $\mu \mathrm{m}$ and 1000$\mu \mathrm{m}$ dimension filters developed considerable mirror bowing and exhibited total transmission degradation with actuation. The effects of top mirror bowing on the on-pixel spectral performance of the 500- $\mu \mathrm{m}$ and $1000-\mu \mathrm{m}$ dimension filters were examined. It was shown that although these two filters can achieve ideal on-pixel transmission characteristics, they suffer from significant pixel-to-pixel transmission peak wavelength variations and, as a result, have narrower usable spectral tuning ranges in comparison to the $200-\mu \mathrm{m}$ dimension filter; namely, 9.3-11.4 $\mu \mathrm{m}$ and $10.3-11.3 \mu \mathrm{m}$ for the 500- $\mathrm{mm}$ and 1000$\mu \mathrm{m}$ dimension filters, respectively. Overall, we anticipate the work presented in this paper to be a good starting point for the future development of miniature multispectral thermal imaging systems based on a hybridized technology incorporating a large-area tunable LWIR Fabry-Perot filter and a 2-D LWIR imaging focal plane array. The MEMS-based LWIR FabryPerot filters presented in this paper have been designed to be operated at liquid nitrogen temperature, as required for 2-D LWIR imaging focal plane arrays. Cryogenic cooling tests have been conducted on the fabricated filters (not shown here), and preliminary results demonstrate that the filters can maintain excellent nanometre-scale mirror flatness even after cooling to $77 \mathrm{~K}$. However, at present, our cryogenic testing setup is not capable of measuring electrical actuation and optical transmission simultaneously. Thus, the optical tuning behaviour of the cooled filters will be undertaken as part of future research.

\section{REFERENCES}

[1] L. Zhang and G. W. Small, "Automated detection of chemical vapors by pattern recognition analysis of passive multispectral infrared remote sensing imaging data," Appl. Spectrosc., vol. 56, no. 8, pp. 1082-1093, Feb. 2002.

[2] S. Teggi, M. P. Bogliolo, M. F. Buongiorno, S. Pugnaghi, and A. Sterni, "Evaluation of $\mathrm{SiO}_{2}$ emission from Mount Etna using diurnal and nocturnal multispectral IR and visible imaging spectrometer thermal IR remote sensing images and radiative transfer models," J. Geophys. Res.: Sol. Ea., vol. 104, no. B9, pp. 20069-20079, Sep. 1999.

[3] M. J. Wabomba, Y. Sulub, and G. W. Small, "Remote detection of volatile organic compounds by passive multispectral infrared imaging measurements," Appl. Spectrosc., vol. 61, no. 4, pp. 349-358, Apr. 2007.

[4] A. B. Kahle and A. F. H. Goetz, "Mineralogic information from a new airborne thermal infrared multispectral scanner," Science, vol. 222, no. 4619, pp. 24-27, Oct. 1983.

[5] R. G. Vaughan, S. J. Hook, W. M. Calvin, and J. V. Taranik, "Surface mineral mapping at Steamboat Springs, Nevada, USA, with multi-wavelength thermal infrared images," Remote Sens. Environ., vol. 99, no. 1-2, pp. 140-158, Nov. 2005.

[6] W. B. Clodius, C. Borel, L. Balick, and S. J. Hook, "Validation of the MTI water surface temperature retrieval algorithms," in Proc. IEEE Int. Geosci. Remote Sens. Symp. (IGARSS'02), 2002, pp. 30-32.

[7] A. P. Rodger, L. K. Balick, and W. B. Clodius, "The performance of the multispectral thermal imager (MTI) surface temperature retrieval algorithm at three sites," IEEE Trans. Geosci. Remote Sens., vol. 43, no. 3, pp. 658-665, Mar. 2005.

[8] H. A. Macleod, "Multilayer high-reflectance coatings," in Thin-film Optical Filters, W. T. Welford, Ed., 3rd ed. Bristol and Philadelphia: Institute of Physics Publishing, 2001, ch. 5, sec. 1, pp. 179-183.

[9] P. A. Stupar, R. L. Borwick, J. F. DeNatale, P. H. Kobrin, and W. J. Gunning, "MEMS tunable Fabry-Perot filters with thick, two sided optical coatings," in Proc. IEEE 15th Int. Conf. Solid-State Sens., Actuators, Microsyst. (TRANSDUCERS'09), Denver, CO, 2009, pp. 1357-1360.

[10] M. Ebermann, M. Meinig, S. Kurth, K. Hiller, E. Gittler, and N. Neumann, "Tiny mid- and long-wave infrared spectrometer module with a MEMS dual-band Fabry-Perot filter," in Proc. Sensor+Test Conf.: IRS ${ }^{2}$, Nürnberg, 2011, pp. 94-99.

[11] M. Meinig, M. Ebermann, N. Neumann, S. Kurth, K. Hiller, and T. Gessner, "Dual-band MEMS Fabry-Perot filter with two movable reflectors for mid- and long-wave infrared microspectrometers," in Proc. IEEE 16th Int. Conf. Solid-State Sens., Actuators, Microsyst. (TRANSDUCERS'11), Beijing, China, 2011, pp. 2538-2541.

[12] M. Tuohiniemi, A. Nasila, and J. Makynen, "Characterization of the tuning performance of a micro-machined Fabry-Perot interferometer for thermal infrared," J. Micromech. Microeng., vol. 23, no. 7, pp. 1-7, Jul. 2013.

[13] M. Tuohiniemi, M. Blomberg, A. Akujarvi, J. Antila, and H. Saari, "Optical transmission performance of a surface-micromachined FabryPerot interferometer for thermal infrared," J. Micromech. Microeng., vol. 22, no. 11, pp. 1-7, Sep. 2012.

[14] J. M. Vaughan, "The plane Fabry-Perot interferometer," in The FabryPerot Interferometer, E. R. Pike and W. T. Welford, Eds. Bristol and Philadelphia: Adam Hilger, 1989, ch. 3, sec. 6, pp. 123-127.

[15] G. J. Sloggett, "Fringe broadening in Fabry-Perot interferometers," Appl. Opt., vol 23, no. 14, pp. 2427-2432, Jul. 1984.

[16] H. Mao, A. J. Keating, K. K. M. B. D. Silva, J. M. Dell, and L. Faraone, "Long-wavelength infrared Fabry-Perot etalon for multi-spectral thermal imaging," in Proc. SPIE, vol. 8923, pp. 89234-89239, Dec. 2013.

[17] H. A. Macleod, "Basic theory," in Thin-film Optical Filters, W. T. Welford, Ed., 3rd ed. Bristol and Philadelphia: Institute of Physics Publishing, 2001, ch. 2, sec. 5, pp. 43-45.

[18] R. F. Potter, "Germanium (Ge)," in Handbook of Optical Constants of 
Solids, vol. 1, E. D. Palik, Ed. Burlington: Elsevier, 2012, ch. 17, pp. 475-476.

[19] E. D. Palik and A. Addamiano, "Zinc sulfide (ZnS)," in Handbook of Optical Constants of Solids, vol. 1, E. D. Palik, Ed. Burlington: Elsevier, 2012, ch. 27, pp. 613-614.

[20] E. Garmire, "Theory of quarter-wave-stack dielectric mirrors used in a thin Fabry-Perot filter," Appl. Opt., vol 42, no. 27, pp. 5442-5449, Sep. 2003.

[21] W. S. Lau, "The measurement of oxygen and carbon and other impurities in silicon," in Infrared Characterization for Microelectronics. Singapore: World Scientific, 1999, ch. 3, pp. 25-30.

[22] C. Roychoudhuri and M. Hercher, "Stable multipass Fabry-Perot interferometer: design and analysis," Appl. Opt., vol 16, no. 9, pp. 2514-2520, Sep. 1977. 